stomatologie $2013 \cdot 110: 32-32$

DOI 10.1007/s00715-013-0217-1

C Springer-Verlag Wien 2013

\section{H. Haider}

Österreichische Gesellschaft für Zahn-, Mund- und Kieferheilkunde, Markt Allhau

\title{
Neue Rubrik: Wie löse ich diesen Fall?
}

Sehr geehrte Frau Kollegin, sehr geehrter Herr Kollege!

Wie löse ich diesen Fall? Mit Sicherheit hat sich jeder von uns im Praxisalltag diese Frage schon oft gestellt. Die neue Rubrik „Wie löse ich diesen Fall?“, deren Idee wir Frau Dr. Corinna Bruckmann verdanken, verfolgt mehrere Ziele.

Jeder von uns wurde im Praxisalltag schon öfters mit Problemen konfrontiert, die uns an die Grenzen unseres Wissens und Könnens geführt haben.

Jeder von uns hat im Praxisalltag schon Fälle erlebt, die so interessant waren, dass wir wohl gedacht haben, es wäre doch interessant, diesen Fall in einem größeren Rahmen Kolleginnen und Kollegen präsentieren zu können.

\section{》) Aus der Praxis für die Praxis}

Jeder von uns hat zumindest in Fortbildungsveranstaltungen, bei Kongressen, im Internet, in der Fachliteratur usw. Fälle kennengelernt, die es wert gewesen wären, einem größeren Publikum zugänglich gemacht zu werden.

Das deklarierte Ziel der neuen Rubrik „Wie löse ich diesen Fall“ ist es, für solche interessante, nicht alltägliche Prob-

\section{Schreiben Sie uns}

Anregungen, Themenvorschläge, Wünsche und Kritik zur Rubrik,,Wie löse ich diesen Fall?" richten Sie bitte an:

Prof. Dr. H. Haider

Gemeindestr. 29/5

7411 Markt Allhau

herbert.haider@live.at lemstellungen aus dem Praxisalltag eine Plattform zu bilden - eben „aus der Praxis für die Praxis“.

Solche Fälle können durchaus schockierend sein, manchmal werden sie „nur" hochinteressant sein, manche werden polarisieren und manchmal könnten sie direkt aus der „Schmunzelecke" stammen.

Damit die Rubrik aber auch ein wirklicher Erfolg werden kann, müssen mehrere Parameter gegeben sein:

Wir brauchen zunächst Kolleginnen und Kollegen, die bereit sind, uns solche Fälle zu melden.

Wir brauchen aber auch Kolleginnen und Kollegen, die den Mut aufbringen, ihre Diagnose und den Therapievorschlag öffentlich zu präsentieren.

Wir brauchen aber auch Ihr Echo, liebe Kolleginnen und Kollegen. Teilen Sie uns einfach Ihre Meinung zu den gezeigten Fällen und den angebotenen Lösungsansätzen mit. Wie hätten Sie therapiert?

\section{\) Das Echo der Leser ist gefragt}

Ich denke, Diagnose und Therapie sind nur selten eine Einbahnstraße. Möglicherweise führen mehrere Wege zum Ziel. Schon der erste, in diesem Heft präsentierte Fall zeigt mir aber auch, dass Dogmen in der modernen zahnärztlichen Therapie nicht unumstößlich sind.

Nun ist Ihre geschätzte Meinung gefragt. Ihr Pro und Kontra zu dieser neuen Rubrik macht unsere neue stomatologie wieder eine Nuance interessanter für uns alle, nach dem Motto „Aus der Praxis für die Praxis“.

Für Ihr Engagement bedankt sich

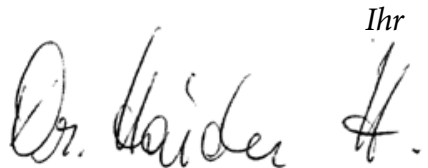

Prof. Dr. Herbert Haider

Präsident

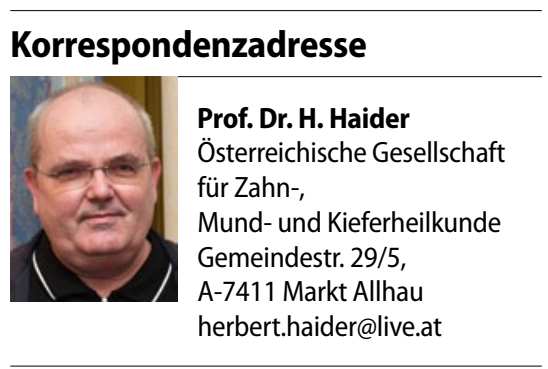

